\begin{tabular}{|c|c|}
\hline Title & Shape Optimization of Double A ntenna for Long Range Passive UHF-Band RFID \\
\hline Author(s) & W atanabe, Y uta; W atanabe, Kota; Igarashi, Haj ime \\
\hline Citation & $\begin{array}{l}\text { IEEE T ransactions on Magnetics, 49(5), 2133-2136 } \\
\text { https://doi.org/10.1109/T MA G.2013.2241752 }\end{array}$ \\
\hline Issue Date & $2013-05$ \\
\hline Doc URL & http:/hdl.handle.net/2115/53099 \\
\hline Rights & $\begin{array}{l}\text { (C) } 2013 \text { IEEE. Personal use of this material is permitted. Permission from IEEE must be obtained for all other uses, in } \\
\text { any current or future media, including reprinting/republishing this material for advertising or promotional purposes, } \\
\text { creating new collective works, for resale or redistribution to servers or lists, or reuse of any copyrighted component of } \\
\text { this work in other works. }\end{array}$ \\
\hline Type & article (author version) \\
\hline File Information & Shape Optimization of Double A ntenna for Long Range Passive UHF-Band RFID.pdf \\
\hline
\end{tabular}

Instructions for use 


\title{
Shape Optimization of Double Antenna for Long Range Passive UHF-band RFID
}

\author{
Yuta Watanabe ${ }^{1}$, Kota Watanabe ${ }^{2}$, and Hajime Igarashi ${ }^{1}$, Member, IEEE \\ ${ }^{1}$ Graduate School of Information Science and Technology, Hokkaido University \\ Kita 14, Nishi 9, Kita-ku Sapporo, 060-0814, JAPAN \\ ${ }^{2}$ Department of Information and Electronic, Muroran Institute of Technology
}

\begin{abstract}
The passive RFID tag, which receives energy for its operation from electromagnetic waves radiated by RFID readers, changes the antenna impedance to modulate the back scattering waves caught by the reader. For this reason, during the back-scattering communication, the energy received by the RFID tag much reduces due to the impedance mismatch. To resolve this problem, the double antenna system which consists of receiving and sending antennas has been proposed. In this paper, the double antenna shapes are optimized on the basis of FDTD analysis and genetic algorithm to maximize the communication distance.
\end{abstract}

Index Terms-Passive UHF-band RFID, FDTD, MNA, shape optimization.

\section{INTRODUCTION}

$\mathrm{R}$ ADIO FREQUENCY IDENTIFICATION (RFID) has attained considerable attentions [1], [2]. Especially, the UHF-band passive RFID has intensively been studied due to its future applications to, e.g., security and wireless sensing systems [3][6]. The UHF-band passive RFID system consists of a reader and several RFID tags. The reader sends power and data to RFID tags by electromagnetic waves. The RFID tag composed of an antenna and IC chip receives electromagnetic waves, and then sends back data by modulating backscatter waves.

The long rang communication between the reader and RFID tag is essential for its wide applications. Therefore it is important to keep impedance matching between the RFID antenna and IC chip. The passive RFID tag changes the antenna impedance to modulate the back scattering waves caught by the reader. For this reason, during the backscattering communication, the energy received by the RFID tag much reduces due to the impedance mismatch. To resolve this problem, the double antenna system which consists of receiving and sending antennas has been proposed [7]. Each antenna in this RFID tag performs either receiving or backscattering. The receiving antenna continuously receives electromagnetic waves sent from the reader without the impedance modulation. The back-scattering antenna reflects the modulated waves back to the reader.

Although it has been revealed in [7] that the double antenna system which consists of half and 1/4 wavelength dipoles has good performance, its performance would be more improved by optimizing the antenna shapes. In this paper, we present shape optimization of the double antenna taking coupling between the non-liner IC chip and electromagnetic waves into account. In this study, the RFID tags are computed by the finite-difference time-domain (FDTD) method coupled with modified nodal analysis (MNA) [6], [8], [9]. The shape of the double antenna is optimized by the micro genetic algorithm

Manuscript received October 31, 2012 (date on which paper was submitted for review). Corresponding author: Y. Watanabe (e-mail: ywata@em-si.eng.hokudai.ac.jp).

Digital Object Identifier inserted by IEEE
( $\mu$-GA) which mimics the process of natural evolution [10], [11]. The shape optimization considering multi-antenna has not been presented. This method can be applied to optimization of antenna shape considering electromagnetic coupling between antenna and scattering substance.

This paper is organized as follows: in section II, the structure of the RFID tag composed of the double antenna and Cockcroft-Walton (CW) circuit will be described. In section III, the hybridization of the FDTD method and MNA will be formulated and the algorithm of $\mu$-GA will be described. In section IV, the optimization problems and results will be described and discussed.

\section{StRUCTURE OF RFID TAG}

Figure 1 shows the structure of the RFID tag composed of the double antenna and IC chip. The voltage multiplier amplifies and rectifies the input voltage induced by the receiving antenna. The digital section creates the modulating signals composed of ID, sensing data and so on. The backscatter modulator consists of two variable capacitance diodes $D$ and resistors $R_{1}, R_{2}$ and $R_{3}$. Resistors $R_{1}$ and $R_{2}$ keep the voltage of the back-scattering antenna to $V_{\text {. }}$. The resistor $R_{3}$ separates the back-scattering antenna to the digital section. The characteristics of the variable capacitance diode vary with respect to the applied voltage. The input impedance seen from the back-scattering antenna is varied by the modulating signals.

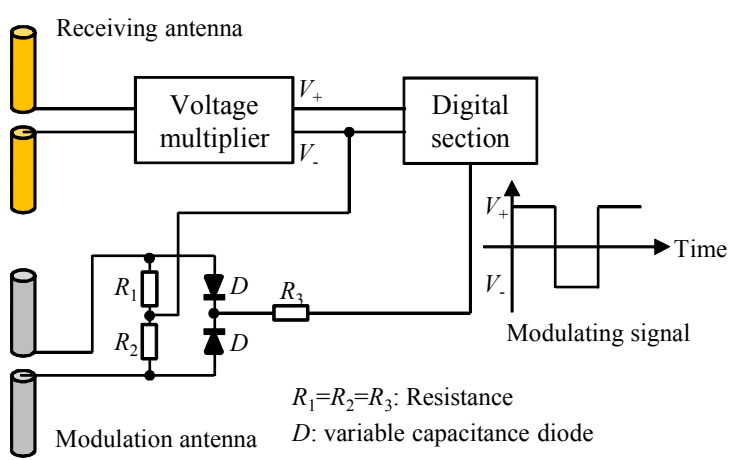

Fig. 1. Structure of RFID tag composed of the double antenna and IC chip. 


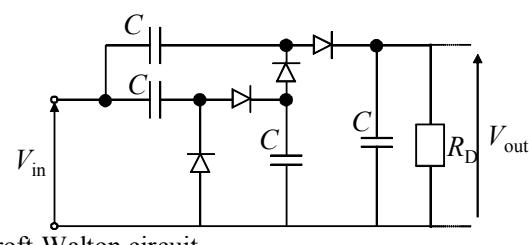

Fig. 2. Cockcroft-Walton circuit.

In this study, the receiving and back-scattering antennas are assumed to be the meander line antenna (MLA) and line antenna. The CW circuit shown in Fig. 2 is employed for the voltage multiplier. The digital section is represented by resistance $R_{\mathrm{D}}$ for simplicity. The back-scattering antenna and backscatter modulator are assumed to be isolated from the receiving antenna, voltage multiplier and digital section.

\section{OPTIMIZATION METHOD}

\section{A. Hybridization of FDTD Method and MNA}

To take non-linearity of diodes in the $\mathrm{CW}$ circuit into consideration, we perform time-domain analysis of electromagnetic fields coupled with the non-liner circuit. The Maxwell equations are discretized with the central finite differences for space and time as follows:

$$
\begin{aligned}
& \boldsymbol{E}^{n}=\frac{1-\sigma \Delta t / 2 \varepsilon}{1+\sigma \Delta t / 2 \varepsilon} \boldsymbol{E}^{n-1}+\frac{\Delta t / \varepsilon}{1+\sigma \Delta t / 2 \varepsilon} \nabla \times \boldsymbol{H}^{n-1 / 2} \\
& \boldsymbol{H}^{n+1 / 2}=\boldsymbol{H}^{n-1 / 2}+\frac{\mu}{\Delta t} \nabla \times \boldsymbol{E}^{n-1}
\end{aligned}
$$

where $n$ denotes time step. In the FDTD method, $\boldsymbol{E}^{n}$ and $\boldsymbol{H}^{n+1 / 2}$ are calculated alternately.

MNA determines the node voltage vector $v$ in the non-liner circuit from Kirchhoff's laws. The nodal equation for the nonliner circuit is given by

$$
f(v)+\mathrm{C} \frac{\mathrm{d} v}{\mathrm{~d} t}=\boldsymbol{i}
$$

where $\boldsymbol{i}$ is the current source vector of the non-linear circuit, $\boldsymbol{f}$ is the non-linear function which expresses the current determined from the resistance and active devices such as the diode, and $\mathrm{C}$ is the matrix representing capacitance [6]. In this work, (2) is solved using the backward Euler method and the Newton-Raphson method.

To couple the FDTD method with MNA, let us consider the line antenna loaded by the non-liner circuit shown in Fig. 3. The spatial size of the non-liner circuit is assumed to be sufficiently smaller than the size of FDTD cell. By integrating Ampere' law on the surface $S$ of FDTD cell, we obtain

$$
C_{0} \frac{\mathrm{d} V_{\mathrm{L}}}{\mathrm{d} t}+I_{\mathrm{L}}=I
$$

where $V_{\mathrm{L}}=E_{\mathrm{Z}} \Delta Z$ is the voltage imposed to the non-liner circuit, $C_{0}=\varepsilon_{0} \Delta X \Delta Y / \Delta Z$ is the spatial capacitance of the FDTD cell, $S$ $=\Delta X \Delta Y$ is the area of the FDTD cell, $\Delta Z$ is the height of the

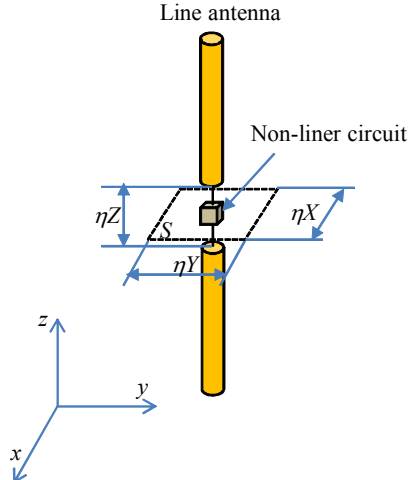

Fig. 3. Line antenna loaded by non-liner circuit. $\Delta X, \Delta Y$ and $\Delta Z$ are size of the FDTD cell.

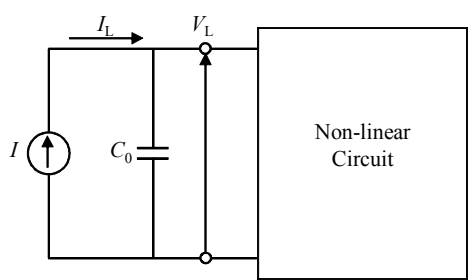

Fig. 4. Equivalent circuit of the coupling equation.

FDTD cell, $I_{\mathrm{L}}$ is the current flowing into the non-liner circuit, and $I$ is the total current given by $I=\int_{\partial S} \boldsymbol{H} \cdot \mathrm{d} \boldsymbol{s}$. Equation (3), which couples the FDTD method with MNA, is included in (2). The equivalent circuit corresponding to (3) is shown in Fig.4, which is composed of a parallel circuit of the total source current $I$, capacitance $C_{0}$ and non-liner circuit. Node voltages of this equivalent circuit are obtained by MNA.

The followings are procedures for the hybridization of the FDTD method and MNA.

1) In the FDTD process, the magnetic field $\boldsymbol{H}^{n-1 / 2}$ is computed.

2) The total source current $I^{n-1 / 2}$ is calculated from the $\boldsymbol{H}^{n-1 / 2}$.

3) $I^{n-1 / 2}$ is substituted to (2), which is then solved by MNA for $V_{\mathrm{L}}^{n}$.

4) The resultant electric field $E_{\mathrm{Z}}{ }^{n}=V_{\mathrm{L}}{ }^{n} / \Delta Z$ is substituted to (1b), which is solved by the FDTD method for $\boldsymbol{H}^{n+1 / 2}$.

5) The calculations of $\boldsymbol{E}, \boldsymbol{H}, I$ and $V_{\mathrm{L}}$ are repeated until steady solution is obtained.

Mur's boundary condition is employed to impose the free space conditions on the domain boundary in the FDTD method. In this study, the V-I characteristic of the diode is assumed to be

$$
I_{D}=\left\{\begin{array}{cc}
1.0 \times 10^{-8} V_{\mathrm{D}} & V_{\mathrm{D}}<0 \\
2.9 \times 10^{-7}\left[\exp \left(15 V_{\mathrm{D}}\right)-1\right] & V_{\mathrm{D}} \geq 0
\end{array}\right.
$$

where $V_{\mathrm{D}}$ is the voltage imposed to the diode.

\section{B. Micro Genetic Algorithm}

In this study, the $\mu-\mathrm{GA}$ is employed to obtain optimal shapes of the double antenna. Because $\mu$-GA has very small population size, it is advantageous for our optimization problem which needs computationally expensive fitness evaluations. The followings are procedures in $\mu-\mathrm{GA}$. 
1) The initial population of size $N_{\text {pop }}$ is generated randomly.

2) The fitness of each individual is evaluated. The fitness is computed through the FDTD analysis coupled with MNA.

3) The individual having the highest fitness is preserved as an elite individual.

4) Two individuals are selected randomly as parents by performing tournament selection.

5) A child is produced by applying BLX- $\alpha$ to the parents. In this study, $\alpha$ is set to 0.3 .

6) $N_{\text {pop }}-1$ children are produced by repeating steps 4 and 5 .

7) If the population converges, it is reinitialized.

8) Steps 2 to 7 are repeated until the iteration number reaches to maximum.

In the reinitialization, all individuals are newly generated if the population is judged to have converged to local optimum. In the $\mu-\mathrm{GA}$, the reinitialization actualizes the global search. Preservation of the elite individual and selection processes realize local search.

\section{OPTIMIZATION}

\section{A. Optimization Problem}

The double antenna loaded by the $\mathrm{CW}$ circuit shown in Fig. 2 is optimized to maximize the communication distance between the RFID tags and reader. The RFID tags must be able to start operating by receiving weak electromagnetic waves so that the long range communication is realized. Therefore we maximize the output voltage $V_{\text {out }}$ of the CW circuit included in the RFID tag. Figure 5 shows the model of the UHF-band passive RFID for the field analysis. Plane waves are incident to the RFID tag, assuming that the reader is sufficiently far from the RFID tag. We maximize $V_{\text {out }}$ of the $\mathrm{CW}$ circuit when the plane wave is incident on the double antenna under the situation that input impedance of backscattering antenna is changed. The optimization problem is defined by

$$
f=V_{\text {out }}^{\text {open }}(\boldsymbol{x}, \boldsymbol{y})+\alpha V_{\text {out }}^{\text {short }}(\boldsymbol{x}, \boldsymbol{y}) \rightarrow \max
$$

where $\boldsymbol{x}$ and $\boldsymbol{y}$ are the design parameters of the double antenna, and $V_{\text {out }}^{\text {off }}$ and $V_{\text {out }}^{\text {on }}$ are the output voltages of the $\mathrm{CW}$ circuit when the back-scattering antenna terminal is short and open. In the optimization, $\alpha$ is set to 0.8 .

The characteristics of the double antenna, such as the input

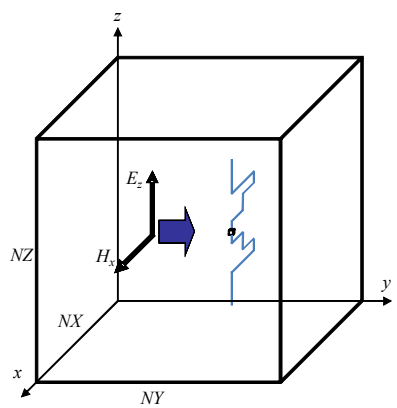

Fig. 5. Analysis model of the UHF-band RFID systems. Plane wave is incident to the RFID tag, assuming that the reader is sufficiently far from the RFID tag. impedance and gain, depend on its shape. The genotype and phenotype in the $\mu$-GA are shown in Fig. 6. The antenna shape is decoded from the gene through the following procedure.

1) A base line is divided into small line segments. In this study, the number of segments is set to five.

2) To form the receiving antenna, each segment is moved in a perpendicular direction to the base line by a distance described in the gene of $\mu-\mathrm{GA}$.

3) Length of each segment is changed by a distance described in the gene.

4) Both ends of each segment are connected to adjacent segments.

5) The other half of received antenna is similarly formed.

6) The modulation antenna is formed out of the line antenna set to horizontal length of receiving antenna.

\section{B. Optimization Results}

In the optimization, the frequency and amplitude of the incident wave are set to $1 \mathrm{GHz}$ and $15 \mathrm{~V} / \mathrm{m}$. In the FDTD processes, the size of FDTD cells, where $\Delta X=\Delta Y=\Delta Z$, is set to $3 \mathrm{~mm}$. The number of FDTD cells, where $N X=N Y=N Z$, is set to 100 . In the MNA process, the capacitances $C$ are set to $1 \mathrm{pF}$, and the resistance $R_{\text {out }}$ is set to $166 \mathrm{k} \Omega$. The computational time of FDTD analysis carried out on intel Xeon 5160 is $744 \mathrm{~s}$.

The optimized shapes of the double antenna obtained by $\mu$ GA are shown in Fig. 7, where (a) and (b) include the MLA and line antenna for the receiving antenna, respectively. When we obtain the double antenna system shown in Figure 7 (b), we optimize the antenna length and distance between receiving and back-scattering antennas. The length of the optimized double antenna (a) is shorter than length of the halfwave dipole antenna, $150 \mathrm{~mm}$, at $1 \mathrm{GHz}$. The time evolution of the output voltages of the $\mathrm{CW}$ circuit which is connected to the optimized double antenna as well as a pair of straight dipole antennas of $1 / 2$ and $1 / 4$ wavelengths, discussed in [7], are shown in Fig. 8 for comparison. It can be seen in Fig. 8 that $V_{\text {out }}$ tends to increase almost monotonously and reach at the steady value although it includes small ripples. It is found that the optimized antennas shown in Fig.7 (a) provides higher output voltage in comparison with the dipole pairs. The input voltage of the $\mathrm{CW}$ circuit depends on the input impedance of antenna. Therefore, optimized antennas keep impedance
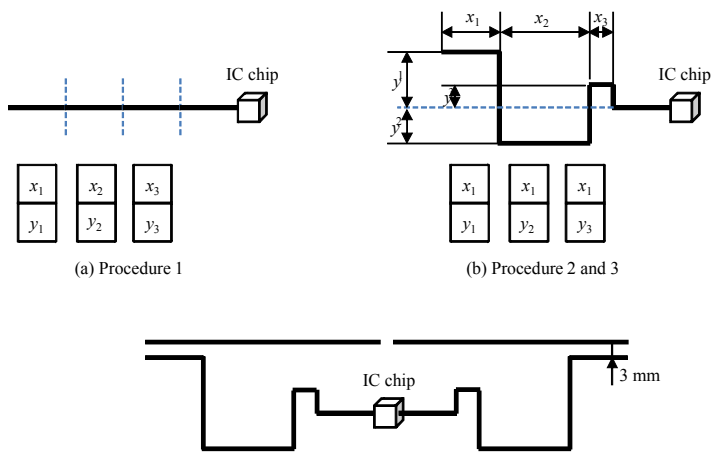

(c) Procedure 4 and 5

Fig. 6. Phenotype and genotype. 
matching between antenna and IC chip impedance when impedance of backscattering antenna is changed.

Maximum output voltages $V_{\text {out }}$ of the $\mathrm{CW}$ circuit with double antenna and modulation degrees are summarized in Table I, where the modulation degree is given by

$m=\frac{\left|\boldsymbol{E}_{\mathrm{s}}\right|}{\left|\boldsymbol{E}_{\mathrm{c}}\right|}=\frac{2\left(\left|\boldsymbol{E}_{\mathrm{bs}}^{\text {short }}\right|-\left|\boldsymbol{E}_{\mathrm{bs}}^{\text {open }}\right|\right)}{\left|\boldsymbol{E}_{\mathrm{bs}}^{\text {short }}\right|+\left|\boldsymbol{E}_{\mathrm{bs}}^{\text {open }}\right|}$

where $\boldsymbol{E}_{\mathrm{c}}, \boldsymbol{E}_{\mathrm{s}}$ and $\boldsymbol{E}_{\mathrm{bs}}$ denote the carrier, signal and backscatter waves shown in Fig. 9. The numerical results show that the optimized shape (a) has higher value for $V_{\text {out }}$ than the optimized shape (b) and pair of dipole antennas. However the optimized shape (a) has lower modulation degree. Therefore, if the optimized shape (a) is employed to the RFID antenna, it is required that the reader has a good ability in demodulation.

\section{CONCLUSIONS}

The shape of MLA for the double antenna system is optimized. The characteristics of the double antenna loaded by
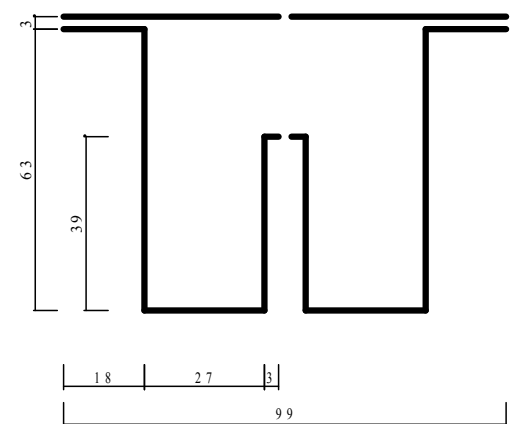

(a) Double antenna composed of MLA and dipole antenna

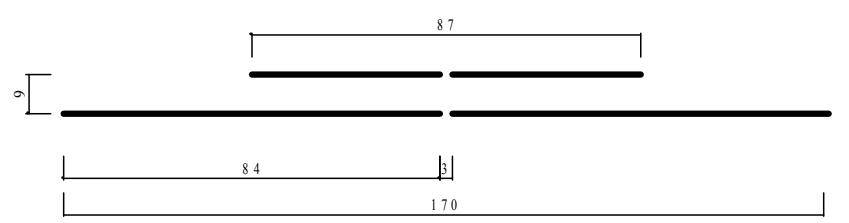

(b) Double antenna composed of a pair of dipole antenna

Fig. 7.Double antenna obtained by $\mu$-GA.

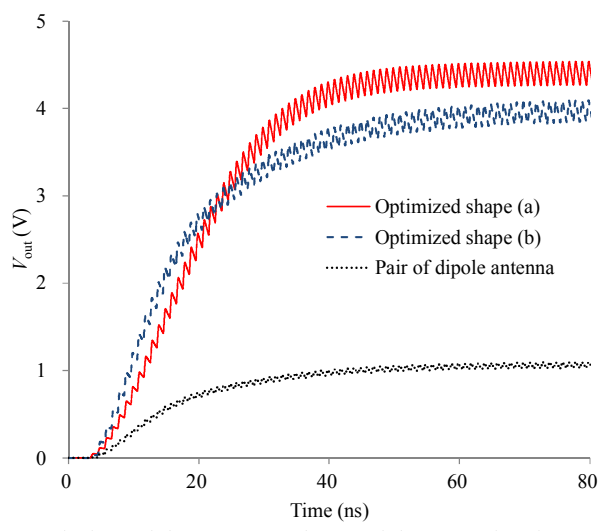

Fig. 8.Time evolution of the output voltage of the $\mathrm{CW}$ circuit.
TABLE I

OUTPUT VOLTAGE OF CW CIRCUIT AND MODULATION DEGREE.

\begin{tabular}{cccc}
\hline \hline & $V_{\text {out }}^{\text {off }}(\mathrm{V})$ & $V_{\text {out }}^{\text {on }}(\mathrm{V})$ & $\begin{array}{c}\text { Modulation } \\
\text { degree (\%) }\end{array}$ \\
\hline Optimized shape (a) & 4.5 & 4.1 & 9.5 \\
Optimized shape (b) & 4.1 & 3.8 & 9.2 \\
Pair of dipole antennas & 1.1 & 0.8 & 21.0 \\
\hline \hline
\end{tabular}

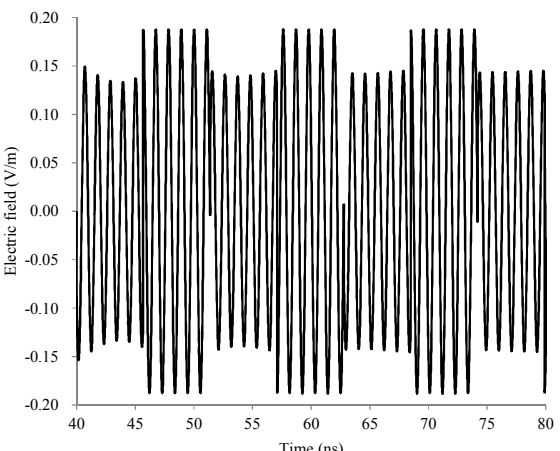

Fig. 9 Time evolution of electromagnetic wave scatted by optimized antenna shown in Fig. 7 (a).

the CW circuit are obtained by the FDTD analysis coupled with MNA. The resultant double antenna obtained by $\mu$-GA, which has relatively small area, has the higher output voltage of the $\mathrm{CW}$ circuit in comparison with the pair of dipole antennas of $1 / 4$ and $1 / 2$ wavelength. However if the double antenna obtained by $\mu$-GA is employed, the reader should have a good ability in demodulation because the modulation degree of the optimization double antenna is relatively small.

\section{REFERENCES}

[1] R. Want, "An introduction to RFID technology," IEEE Pervasive Computing, pp. 25-33, 2006.

[2] A. Juels, "RFID security and privacy: a research survey," IEEE Journal on Selected Areas in Communications, vol. 24, no. 2, pp. 381-394, 2006.

[3] G. Marrocco, "Gain-optimized self-resonant meander line antennas for RFID applications," IEEE Antennas and Wireless Propagation Letters, vol. 2, pp. 302-305, 2003.

[4] K. V. Seshagiri Rao, P. V. Nikitin and S. F. Lam," Antenna design for UHF-band RFID tags: A review and practical application," IEEE Transactions on Antennas and Propagation, vol. 53, no. 12, pp. 38703876, 2005.

[5] H. Makimura, Y. Watanabe, K. Watanabe, and H. Igarashi, "Evolutional design of small antennas for UHF-band RFID," IEEE Trans. Magn., vol. 47, no. 5, pp. 1510-1513, 2011

[6] Y. Watanabe, K. Watanabe, and H. Igarashi, "Optimization of meander line antenna considering coupling between nonlinear circuit and electromagnetic waves for UHF-band RFID," IEEE Trans. Magn., vol. 47, no. 5, pp. 1506-1509, 2011.

[7] K. Watanabe, Y. Watanabe, H. Igarashi, and H. Waki, "Double antenna for passive long range UHF-band RFID," International Journal of Applied Electromagnetics and Mechanics, vol. 39. No. 1-4, pp. 671-676, 2012.

[8] V. A. Thomas, M. E. Jones, M. Piket-May, A. Taflove, and E. Harrigan, "The use of SPICE lumped circuits as sub-grid models for FDTD analysis," IEEE Microwave and Guided Wave Letters, vol. 4, no. 5, pp. 141-143, 1994.

[9] Y. Kanda, and T. Uno, "A few considerations on direct linking of FDTD method and SPICE," Technical Report of IEICE A, pp. 9-15, 1997.

[10] C. A. C. Coello, and G. T. Pulido, "A micro genetic algorithm for multi objective optimization,” EMO 2001, LNCS 1993, pp. 126-140, 2001.

[11] C. T. Pulido, and C. A. C. Coello, "The micro genetic algorithm 2: Toward online adaptation in evolutionary multiobjective optimization," EM 2003, LNCS 2632, pp. 252-266, 2003. 